\title{
Iridium (III) complex-loaded liposomes as a drug delivery system for lung cancer through mitochondrial dysfunction
}

This article was published in the following Dove Press journal: International Journal of Nanomedicine

\section{Cancheng Liao* \\ Danqiao Xu* \\ Xiaohong Liu \\ Yuqi Fang \\ Jun Yi \\ Xiaofang Li \\ Bohong Guo}

Department of Pharmaceutics, Guangdong Pharmaceutical University, Guangzhou 5I0006, China

*These authors contributed equally to this work
Correspondence: Bohong Guo Department of Pharmaceutics, Guangdong Pharmaceutical University, 280 East Waihuan Road, Guangzhou Higher Education Mega Center, Guangzhou 510006, China Email guobohong@gdpu.edu.cn
Background and aim: Iridium (Ir)-based complex is a potential antitumor ingredient, but its poor physicochemical properties such as hydrophobicity and low biocompatibility hamper further application. Liposome provides a potential delivery approach for improving the poor physicochemical property and reducing the side effects of antitumor drug. In this study, we aimed at incorporating $\operatorname{Ir}\left(\left[\operatorname{Ir}(\mathrm{ppy})_{2}(\mathrm{BTCP})\right] \mathrm{PF}_{6}\right)$ into liposomes to enhance the biocompatibility and sustained release of Ir for intravenous administration and to elucidate the mechanism in A549 cells.

Materials and methods: Ir-loaded PEGylated liposomes (Lipo-Ir) were formulated by thin-film dispersion and ultrasonic method. Morphology, size distribution, and zeta potential of Lipo-Ir were examined by transmission electron microscopy (TEM) and Zetasizer. The released profile and biocompatibility were investigated by dialysis method and hemolysis test, respectively. Additionally, the cytotoxic activity and mechanism of Lipo-Ir and Ir inducing apoptosis in A549 cells were evaluated.

Results: Lipo-Ir can keep sustained release, excellent biocompatibility, and physical stability. The average particle size, polydispersity index, zeta potential, encapsulation efficiency, and drug loading are $112.57 \pm 1.15 \mathrm{~nm}, 0.19 \pm 0.02,-10.66 \pm 0.61 \mathrm{mV}, 94.71 \% \pm 3.21 \%$, and $4.71 \% \pm 0.41 \%$, respectively. 3-(4,5-dimethylthiazole)-2,5-diphenltetraazolium bromide (MTT) assay show that Lipo-Ir and Ir display high cytotoxicity against selected cancer cells. Furthermore, the apoptotic features of morphology, depolarization of mitochondrial membrane potential, increase in the reactive oxygen species (ROS) levels, and disorder of $\mathrm{Ca}^{2+}$ homeostasis are observed after treating A549 cells with Ir and Lipo-Ir. Besides, Lipo-Ir can arrest the cell growth in $\mathrm{G}_{0} / \mathrm{G}_{1}$ phase.

Conclusion: The studies demonstrate that Lipo-Ir can trigger apoptosis in A549 cells via ROS-mediated mitochondrial dysfunctions, and the biocompatible and sustained Lipo-Ir will be a promising drug delivery system.

Keywords: iridium complex, liposome, apoptosis, reactive oxygen species, mitochondria

\section{Introduction}

Lung cancer occupies the leading cause of cancer incidence and mortality rates in the USA during the most recent 5 years. ${ }^{1}$ At this point, most patients with lung cancer cannot accept curative surgery, and therefore, chemotherapy is still the major treatment method. Ever since the introduction of cisplatin into the field of oncology, the potential of metal-based anticancer agents had been fully realized and explored. However, cisplatin-based drug exhibited several severe side effects. ${ }^{2,3}$ Therefore, it is inevitable to explore new metal-based agents as a substitution of cisplatin-based drug. Iridium 
(Ir)-based complexes could be great alternatives attributed to their limited side effect, high antitumor activity, and rich photophysical properties. ${ }^{4-7}$ As a promising antitumor active pharmaceutical ingredient (API), Ir (III) complexes can evoke ROS overload, cause a decrease in the mitochondrial membrane potential (MMP), and eventually induce cancer cell apoptosis. ${ }^{8}$

The nonionic surfactants are used as formulation vehicles for many poor soluble anticancer agents such as paclitaxel and docetaxel, but severe hypersensitivity reactions may accompany with the administration. Numerous drawbacks of conventional drug delivery system such as burst release, low biocompatibility, and non-target specificity had emerged in the past. ${ }^{9-13}$ Irregular release may stimulate the fluctuation of drug plasma concentration and pose a potential risk to the patients. Insufficient biocompatibility impairs the physiological capacity of normal cells or tissue. To circumvent these deficiencies, liposomal technology has attracted a great interest in the nanomedicine field owing to the liposomal features of low toxicity, biodegradability, and sustained release. ${ }^{14}$ Liposome is a self-assembled lipid bilayer vesicle through the hydrophobic association of phospholipids. It exists in the shape of unilaminar or multilaminar vesicle that can encapsulate hydrophobic or hydrophilic molecules. ${ }^{15}$ In addition, liposome shows a "stealth" property when the surface was modified with polyethylene glycol (PEG) or PEG-derivative phospholipids, ${ }^{16,17}$ which prevents liposomes from binding most components in blood, especially opsonin. Therefore, the PEGylated liposome can increase stability, reduce the affinity of mononuclear phagocyte system, and extend its half-life. ${ }^{18}$ Due to the presence of a discontinuous endothelial lining in the tumor angiogenesis, PEGylated liposomes can extravert into the interstitial space of vasculature. ${ }^{19}$ Consequently, PEGylated liposomes can passively accumulate in tumor tissues.

Previous reports showed that Ir could induce autophagy and apoptosis of SGC-7901 cells, and exhibited good antineoplastic activity. ${ }^{20}$ However, it was difficult to prepare pharmaceutical formulations for parenteral administration due to the poor physicochemical property of Ir complex. Hence, to ameliorate these shortcomings, we incorporated Ir into lipid bilayer to form an Ir-loaded PEGylated liposomes (Lipo-Ir) and then evaluated the sustained profile and biocompatibility of Ir and Lipo-Ir. Additionally, the anticancer activity of Lipo-Ir was performed against A549 cells. The cytotoxicity in vitro was examined by 3-(4,5dimethylthiazole)-2,5-diphenltetraazolium bromide (MTT) method, and anticancer activity was studied by apoptosis, cellular uptake, intracellular localization, reactive oxygen species (ROS), MMP, intracellular $\mathrm{Ca}^{2+}$ measurement, and cell cycle arrest. Our findings offer promise for the drug delivery system of Lipo-Ir (Scheme 1).

\section{Materials and methods Materials}

Ir was prepared according to the study by Zhang et al. ${ }^{20}$ DSPE-MPEG2000, cholesterol (CHO-HP), and egg yolk lecithin (PC-98T) were purchased from Shanghai A.V.T. Pharmaceutical Ltd (Shanghai, China) (pharmaceutical injection grade, purity $>98 \%$ ). RPMI 1640 was obtained from Thermo Fisher Scientific (Waltham, MA, USA). A549 cells (human lung adenocarcinoma) were purchased from the American Type Culture Collection (ATCC) (Manassas, VA, USA). Fetal bovine serum (FBS) was obtained from Thermo Fisher Scientific (Waltham, MA, USA). All other chemicals and reagents were analytical or chromatographic grade.

\section{Preparation of Lipo-Ir}

Lipo-Ir was prepared using thin-film dispersion method as previously reported with minor modification. ${ }^{21}$ Briefly, PC-98T, CHO-HP, DSPE-MPEG2000, and vitamin E at a molar ratio of 79.7:15.6:3.98:0.7 were dissolved in a mixture of chloroform-alcohol $(3: 1, \mathrm{v} / \mathrm{v})$. Then, Ir (Ir:PC$98 \mathrm{~T}=1: 12.4, \mathrm{w} / \mathrm{w})$ was added to the flask. To form the homogeneous thin film, organic solvent was removed with a rotary vacuum evaporator at $46^{\circ} \mathrm{C}$ and the residual solvent was totally removed under vacuum for $2 \mathrm{~h}$ at $45^{\circ} \mathrm{C}$. Subsequently, the dried lipid film was hydrated with $5 \mathrm{~mL}$ phosphate-buffered saline (PBS; $\mathrm{pH} 7.4$ ) by vortex shedding for $20 \mathrm{~min}$ at $45^{\circ} \mathrm{C}$. To obtain a homogeneous size, the suspensions were sonicated in cell sonicator for $150 \mathrm{~s}$ at $300 \mathrm{~W}$, and then filtered through $200 \mathrm{~nm}$ pore size polycarbonate membranes (EMD Millipore, Billerica, MA, USA). The final products were obtained by freeze-drying. The blank PEGylated liposomes (Blank-Lipo) were prepared using the same procedure without Ir.

\section{Characterization of liposomes}

The morphology of Lipo-Ir was characterized through a transmission electron microscopy (TEM) with negative staining by $3 \%$ phosphotungstic acid after the Lipo-Ir was diluted 10-fold. The particle size, polydispersity index (PDI), and zeta potential of the liposomes were analyzed using a Delsa Nano S Particle Analyzer (Beckman Coulter, Brea, CA, USA) at ambient temperature after diluting to appropriate concentration. 


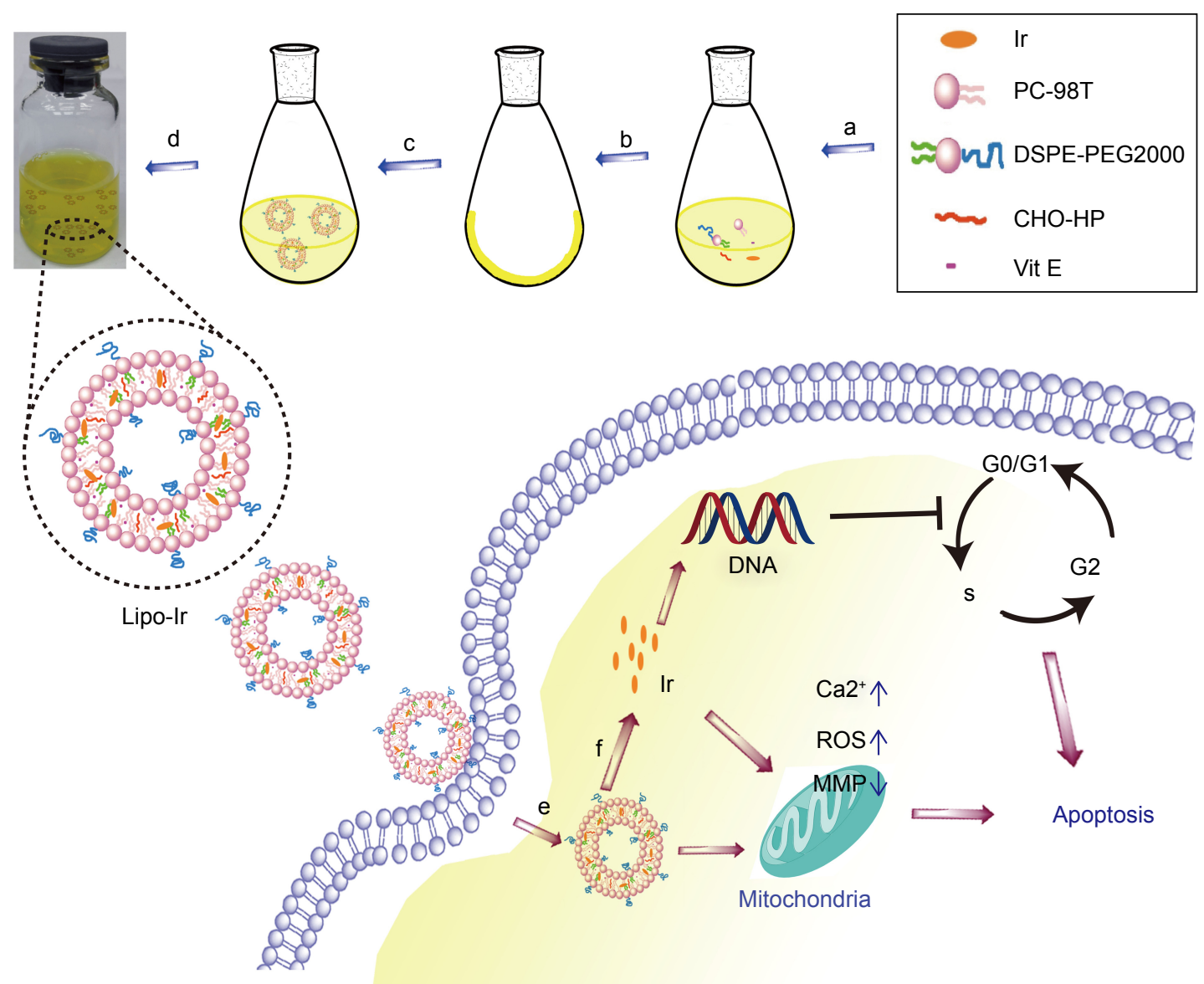

Scheme I Schematic design of Lipo-Ir: (a) mixture and dissolution of material; (b) evaporation of solvent under vacuum to form a lipid film; (c) hydration with PBS and selfassembly; (d) ultrasonic to reduce the diameter; (e) internalization; and (f) Ir release from Lipo-Ir to target mitochondria.

Abbreviations: Ir, iridium; Lipo-Ir, Ir-loaded PEGylated liposomes; PBS, phosphate-buffered saline; PEG, polyethylene glycol; ROS, reactive oxygen species; Vit, vitamin; MMP, mitochondrial membrane potential.

\section{Encapsulation efficiency (EE) and drug loading (DL) of liposomes}

The EE and DL of the liposomes were detected by highvelocity centrifugal method. The resulting liposomes were centrifuged at $35,000 \mathrm{rpm}$ for $1 \mathrm{~h}$ at $4^{\circ} \mathrm{C}$ in a preparative ultracentrifuge. Then, the supernatant containing free Ir was measured by HPLC and the total content of Ir in liposomes suspension was determined after destroying liposomes with ethanol. The EE (\%) and DL (\%) of Lipo-Ir were calculated using the following equations: ${ }^{22}$

$$
\begin{gathered}
\operatorname{EE}(\%)=\frac{M_{\mathrm{t}}-M_{\mathrm{f}}}{M_{\mathrm{t}}} \times 100 \\
\operatorname{DL}(\%)=\frac{M_{\mathrm{t}}-M_{\mathrm{f}}}{M_{\mathrm{t}}-M_{\mathrm{f}}+M_{\mathrm{p}}} \times 100
\end{gathered}
$$

where $M_{\mathrm{f}}$ is the analyzed weight of unentrapped $\mathrm{Ir}$ in the outer aqueous phase, $M_{\mathrm{t}}$ is the total weight of Ir used in the formulation, and $M_{\mathrm{p}}$ is the total weight of lipids added in the formulation.

\section{Drug release from liposomes}

In vitro release profile of Ir from Lipo-Ir suspension was performed using dialysis method. Briefly, Lipo-Ir formulation (300 $\mu \mathrm{g}$ Ir-equivalent) was sealed in cellulose dialysis bags (MWCO $=8-14 \mathrm{kDa}$ ) and immersed in $100 \mathrm{~mL}(\mathrm{pH} 7.4) \mathrm{PBS}$ containing $0.5 \%$ Tween $80(\mathrm{w} / \mathrm{v})$ to maintain sink condition. Afterward, the flask was kept on continuous stirring at $100 \mathrm{rpm}$ using a magnetic stirrer at $37^{\circ} \mathrm{C}$. At predetermined time, aliquot of dialysate $(2 \mathrm{~mL})$ was withdrawn and equivalent volume of fresh buffer was added. The amount of Ir in dialysate was quantified by HPLC, and the cumulative release was calculated. As a contrast, in vitro release of Ir (Ir dispersed in PBS containing $0.5 \%$ Tween $80[\mathrm{w} / \mathrm{v}])$ was implemented as mentioned earlier.

\section{Physical stability of Lipo-Ir}

The physical stability of Lipo-Ir was assessed by monitoring the particle size and EE of Lipo-Ir. Briefly, Lipo-Ir was stored 
in refrigerator $\left(4^{\circ} \mathrm{C}\right)$. After $0,15,30$, and 60 days, Lipo-Ir was diluted appropriately and the particle size was analyzed by DelsaNano S Particle Analyzer. Moreover, the EE of Lipo-Ir was determined by using high velocity centrifugal method as above-mentioned process.

\section{Hemolysis test}

Hemolytic activity of Lipo-Ir was evaluated by measuring the hemolysis percentages of red blood cells (RBCs). ${ }^{23}$ The venous blood was obtained from New Zealand rabbit. All procedures were approved by the GuangDong Pharmaceutical University Experimental Animal Ethics Committee and were performed in accordance with the NIH guidelines for the Care and Use of Laboratory Animals. The RBCs were harvested by centrifugation at $900 \mathrm{rpm}$ for $5 \mathrm{~min}$ and washed several times with normal saline. Afterward, RBCs were resuspended in normal saline at a cell density of $2 \%$ $(\mathrm{v} / \mathrm{v})$. Equivalent volume of $2 \% \mathrm{RBC}$ suspension was mixed with Lipo-Ir suspension to achieve a final Ir concentrations ranging from 18.75 to $93.75 \mu \mathrm{g} / \mathrm{mL}$ and incubated at $37^{\circ} \mathrm{C}$ for $2 \mathrm{~h}$. In addition, RBCs were added to water and normal saline under the same condition as 100 and $0 \%$ hemolysis, respectively. Subsequently, the supernatants were collected by centrifugation at $900 \mathrm{rpm}$ for $5 \mathrm{~min}$. The absorption values of supernatants were measured by an UV-visible light spectrophotometer at $540 \mathrm{~nm}$. For comparison, free Ir was formulated in $4.5 \%$ ethanol $(\mathrm{v} / \mathrm{v})$ and $12 \mathrm{mg} / \mathrm{mL}$ Tween 80 mixed saline solution. Then, the hemolytic activity of $\mathrm{Ir}$ was assessed as process mentioned earlier. The calculation formula of hemolysis percentages was as follows:

$$
\text { Hemolysis }(\%)=\frac{A_{\text {sample }}-A_{0 \%}}{A_{100 \%}-A_{0 \%}} \times 100
$$

where $A_{\text {sample }}, A_{0 \%}$, and $A_{100 \%}$ are the absorbance of samples, a solution of $0 \%$ hemolysis, and a solution of $100 \%$ hemolysis, respectively.

\section{Cytotoxic activity evaluation in vitro}

MTT assay was performed to evaluate the in vitro cytotoxic activity of Ir and Lipo-Ir in A549 cells. The cells were seeded in 96-well microassay culture plates $\left(1 \times 10^{4}\right.$ cells/ well) and incubated at $37^{\circ} \mathrm{C}$ for overnight. The cells treated with Ir (0.47-15 $\mu \mathrm{M}$; final DMSO was $<0.5 \%)$, Lipo-Ir (0.47-15 $\mu \mathrm{M}$ Ir-equivalent), or Blank-Lipo (lipid concentration of $9.11-214.50 \mu \mathrm{g} / \mathrm{mL}$ ) were allowed to incubate for 24 and $48 \mathrm{~h}$ and treated with RPMI 1640 medium as blank control. Upon completion of the incubation, the cells were incubated in serum deprivation medium containing $0.5 \%$ MTT for $4 \mathrm{~h}$. Subsequently, the medium was replaced with $100 \mu \mathrm{L}$ of DMSO to dissolve the formazan. The absorbance was measured at $490 \mathrm{~nm}$ in a microplate spectrophotometer, and the experiments were repeated three times. For the viability of A549 cells, the following formula can be used:

$$
\text { Viability of cells }(\%)=\frac{\text { Absorbance }_{\text {sample }}}{\text { Absorbance }_{\text {control }}} \times 100
$$

\section{Cellular uptake}

A549 cells were placed in 12-well plates at a seeding density of $1.5 \times 10^{5}$ cells/well and incubated overnight. Cells were then exposed to either free Ir or Lipo-Ir at a concentration of $8 \mu \mathrm{M}$ for 0.5 or $2 \mathrm{~h}$. After incubation, the cells were stained with Hoechst 33342 for $10 \mathrm{~min}$ and rinsed twice with cold PBS. The cells were imaged under the ImageXpress ${ }^{\circledR}$ High Content Screening System (IXM; Molecular Devices LLC, Sunnyvale, CA, USA) using $40 \times$ objectives with DAPI and FITC filters. ${ }^{24}$

\section{Intracellular localization}

A549 cells were incubated in 12-well plates at a density of $1.5 \times 10^{5}$ cells/well. After $24 \mathrm{~h}$, cells were incubated with free $\operatorname{Ir}(3 \mu \mathrm{M})$ and Lipo-Ir ( $3 \mu \mathrm{M}$ Ir-equivalent) for $0.5,2$, or $12 \mathrm{~h}$. Afterward, the cells were washed twice using cold PBS and stained with the MitoTracker ${ }^{\circledR}$ Deep Red FM (100 nM; Thermo Fisher Scientific, Waltham, MA, USA) for $\sim 30 \mathrm{~min}$ and rinsed twice with cold PBS again. Fluorescence images of the cells were captured on IXM using $40 \times$ objectives with Texas Red and FITC filters.

\section{Apoptosis morphological changes assay}

The morphology of apoptosis was assessed using acridine orange (AO)/ethidium bromide (EB) and Hoechst 33342 staining methods. Briefly, following incubation of A549 cells in 12-well plates with free Ir or Lipo-Ir ( $3 \mu \mathrm{M}$ Ir-equivalent) for $24 \mathrm{~h}$, the medium was removed and the cells were incubated in FBS-free RPMI 1640 containing AO $(50 \mu \mathrm{g} / \mathrm{mL})$ and $\mathrm{EB}(50 \mu \mathrm{g} / \mathrm{mL})$ for $10 \mathrm{~min}$. After incubation, the cells were rinsed with cold PBS and imaged by IXM.

\section{$\operatorname{MMP}(\Delta \Psi \mathrm{m})$ assay}

A549 cells were treated for $24 \mathrm{~h}$ with $3 \mu \mathrm{M}$ of the Ir and Lip-Ir in 12-well plates and were then washed three times with cold PBS. The cells were incubated for 20 min with $1 \mu \mathrm{g} / \mathrm{mL}$ of $5,5^{\prime}, 6,6^{\prime}$-tetrachloro-1,1',3,3'-tetraethylbenzimidazolcarboc yanine iodide $(\mathrm{JC}-1)$ in culture medium at $37^{\circ} \mathrm{C}$. The cells 
were rinsed with ice-cold PBS again and then were imaged by IXM. The ratio of red/green fluorescence intensity was analyzed by the MateXpress6 software.

\section{ROS detection}

A549 cells were seeded into 12 -well plates at a density of $10 \times 10^{4}$ cells/well and incubated for $24 \mathrm{~h}$. Then, A549 cells were exposed to free $\operatorname{Ir}(3 \mu \mathrm{M})$, Lipo-Ir ( $3 \mu \mathrm{M}$ Ir-equivalent), or equal volume of PBS (negative control group) for $24 \mathrm{~h}$. Afterward, the cells were stained with $10 \mu \mathrm{M} 2^{\prime}, 7^{\prime}$ dichlorodihydrofluorescein diacetate (DCFH-DA) in dark for 20 min. Subsequently, extracellular dye was discarded and cells were rinsed with cold PBS twice. Eventually, images were captured by IXM. The fluorescent intensity was analyzed by the MateXpress6 software.

\section{Intracellular $\mathrm{Ca}^{2+}$ measurement}

Intracellular $\mathrm{Ca}^{2+}\left(\mathrm{Ca}_{\mathrm{i}}^{2+}\right)$ concentration was monitored using Fluo-3 AM as $\mathrm{Ca}^{2+}$ indicator. A549 cells were treated with free $\operatorname{Ir}(3 \mu \mathrm{M})$ and Lipo-Ir $(3 \mu \mathrm{M})$ for $24 \mathrm{~h}$. After treatments, cells were loaded with Fluo-3 AM ( $3 \mu \mathrm{M})$ in dark for $30 \mathrm{~min}$. After washing, the cells were imaged by IXM using $20 \times$ objectives with FITC filter and the fluorescent intensity was analyzed by the MateXpress6 software.

\section{Cell cycle arrest by flow cytometry}

A549 cells were dispensed into six-well plates at a density of $4 \times 10^{5}$ cells/well and incubated for $24 \mathrm{~h}$. Cells were treated with Ir (3 and $5 \mu \mathrm{M}$ ) and Lipo-Ir (3 and $5 \mu \mathrm{M}$ Ir) for $24 \mathrm{~h}$, the cells were harvested by trypsinization, washed with PBS, and fixed with $70 \%$ ethanol at $4^{\circ} \mathrm{C}$ overnight. Then, the fixed cells were collected and resuspended in propidium iodide (PI) staining solution $(20 \mu \mathrm{g} / \mathrm{mL}$ PI, $200 \mu \mathrm{g} / \mathrm{mL}$ RNAse A, and $0.05 \%$ Triton $\mathrm{X}-100$ ) for $30 \mathrm{~min}$ at $37^{\circ} \mathrm{C}$ in dark. Then, the cells were analyzed with a flow cytometer.

\section{Statistical analysis}

The data were evaluated using one-way analysis for statistical significance. The results were presented as mean $\pm \operatorname{SD}(n=3)$. Differences were considered to be statistically significant at $P<0.05$.

\section{Results and discussion Preparation and characterization of liposomes}

Liposomes were first proposed as carriers of biologically active substances in $1971,{ }^{25}$ and it could be used to increase the solubility and biocompatibility of drug and prolong the drug duration in vivo, when targeted specifically. Consequently, we encapsulated Ir into liposomes for intravenous administration. Physicochemical properties of nanoparticles, in some way, determine their behavior in biological application and affect the safety, stability, and effectiveness of colloidal system. ${ }^{26,27}$ Hence, adequate characterization of liposomes is essential. Among the characteristics, surface charge and particle size are the two most commonly mentioned factors, which are associated with biological effects of nanoparticles including cellular uptake, toxicity, and dissolution. ${ }^{26}$ Herein, the particle size, PDI, zeta potential (Figure S1C), EE (\%), and DL (\%) of Lipo-Ir were detected and are listed in Table S1. Additionally, we found that the LipoIr was spherical or quasi-spherical particles (Figure S1B).

The average particle size of Lipo-Ir is $112.57 \pm 1.15 \mathrm{~nm}$, and the PDI value is $0.19 \pm 0.02$, which indicate that Lipo-Ir has a comparatively homogeneous size (Figure S1A). The zeta potential, depending on the kind and composition proportion of lipids, was related with colloid physical stability, because the raise of zeta potential could resist the proximity of particles and promote the stability of dispersion. ${ }^{28}$ BlankLipo and Lipo-Ir showed a negative charge with an average zeta potential up to $-7.03 \pm 0.12$ and $-10.66 \pm 0.61 \mathrm{mV}$, respectively (Table S1). When the absolute value of zeta potential exceeded $30 \mathrm{mV}$, the liposomes were regarded as highly stable, whereas zeta potential in the range of $10-20 \mathrm{mV}$ indicated that the liposomes were relatively stable. ${ }^{29}$ PEGylation was known to facilitate the stability of nanoparticles with the low zeta potential. ${ }^{26}$ Therefore, Lipo-Ir can also exhibit excellent stability in the condition of low zeta potential. In addition, the EE (\%) and DL (\%) of Lipo-Ir were 94.71 and 4.71\%, respectively (Table S1). High EE elucidates that almost all of the Ir can be encapsulated into the liposomes.

\section{Physical stability of liposomes}

During storage, the unstable liposomes tend to aggregate or fuse and then cause size enlargement. Therefore, particle size is an important item to monitor the physical stability of liposomes. When liposomes agglutinate, the particle size of liposome will increase. Also, the decrease in EE will follow the instability of liposomes. As displayed in Table S2, Lipo-Ir possesses high stability in low temperature and no significant changes in particle size and EE are observed in 2 months, which reveals that Lipo-Ir can keep the high physical stability.

\section{In vitro drug release}

The release profile of Lipo-Ir and Ir was studied using the dialysis technique. Lipo-Ir (Figure S2A) displayed a slow 
diffusion rate across the dialysis bag, and the release percentage of Lipo-Ir was only $0.80 \pm 0.09 \%, 14.25 \pm 3.09 \%$, and $47.17 \pm 1.90 \%$ at $0.5,24$, and $96 \mathrm{~h}$, respectively. Obviously, no burst release was observed during the first $30 \mathrm{~min}$. However, the free Ir approximately released $12.11 \pm 6.53 \%$, $47.06 \pm 5.02 \%$, and $91.75 \pm 4.51 \%$ at $0.5,24$, and $96 \mathrm{~h}$, respectively (Figure S2B). Considering the released result, the Lipo-Ir shows significantly sustained release compared with free Ir group. Ir slowly releases from Lipo-Ir, which may be the result of lipid bilayer-induced blockage. Liposomal technology can ameliorate the release profile and circumvent the side effect induced by the irregular drug release.

\section{Hemolysis test}

The first prerequisite for pharmaceutics used for application in vivo is their adequate biocompatibility. Therefore, it is necessary to assess the biocompatibility of formulation for intravenous administration. In this study, we investigated whether or not the Lipo-Ir and Ir result in the rupture of RBCs by hemolysis assay. The hemolytic ratio caused by Ir is $>5 \%$, which is identified as having a risk of hemolysis (Figure S3A). ${ }^{23}$ However, Lipo-Ir does not show any hemolysis when the concentration of Ir reaches $93.75 \mu \mathrm{g} / \mathrm{mL}$, which indicates that Lipo-Ir has a good hemocompatibility and is suitable for potential clinical applications (Figure S3B). The Lipo-Ir, as a drug delivery system, does show much less hemolytic activity than the free Ir complex, which is definitely an advantage of the liposome encapsulation approach.

\section{Cytotoxic activity in vitro}

MTT assay was performed to evaluate the biocompatibility of Blank-Lipo and the in vitro anticancer activity of Lipo-Ir and Ir. As depicted in Figure S4A, when the concentration of empty liposomes reached $214.50 \mu \mathrm{g} / \mathrm{mL}$ (equivalent to Lipo-Ir containing $15 \mu \mathrm{M}$ Ir), significant cytotoxicity was not observed in A549 cells when incubated for 24 or $48 \mathrm{~h}$ (cell viability $>95 \%$ ). The data suggest that the Lipo-Ir can be used for in vivo applications because of their good biocompatibility, ${ }^{30}$ whereas the free Ir and Lipo-Ir show high cytotoxicity against A549 cells in a dose- and time-dependent manner. The half maximal inhibitory concentration $\left(\mathrm{IC}_{50}\right)$ of free Ir was $4.92 \pm 0.22$ and $3.59 \pm 0.21 \mu \mathrm{M}$ at 24 and $48 \mathrm{~h}$, respectively (Figure S4B and $\mathrm{C}$ and Table 1). Lipo-Ir also exhibited a strong cytotoxicity to A549 cells with the $\mathrm{IC}_{50}$ of $6.55 \pm 0.32$ and $3.70 \pm 0.07 \mu \mathrm{M}$ at 24 and $48 \mathrm{~h}$, respectively. The results suggest that Lipo-Ir can retain the antitumor activity of Ir. In addition, the phenomenon that the free Ir displays stronger cytotoxicity than Lipo-Ir during $48 \mathrm{~h}$ may be caused by sustained release of Lipo-Ir.
Table I IC IC $_{50}$ values of Ir and Lipo-Ir formulation toward A549 cell

\begin{tabular}{lll}
\hline Treatment group & $\mathrm{IC}_{50}(\mu \mathrm{M})$ at $24 \mathrm{~h}$ & $\mathrm{IC}_{50}(\mu \mathrm{M})$ at $\mathbf{4 8} \mathbf{h}$ \\
\hline Free $\mathrm{Ir}$ & $4.92 \pm 0.22$ & $3.59 \pm 0.21$ \\
Lipo-Ir & $6.55 \pm 0.32 *$ & $3.70 \pm 0.07$ \\
\hline
\end{tabular}

Notes: Results are presented as the mean \pm SD of triplicates. $* P<0.05$ compared with free Ir.

Abbreviations: $I_{50}$, half maximal inhibitory concentration; Ir, iridium; Lipo-Ir, Irloaded PEGylated liposomes; PEG, polyethylene glycol.

\section{Apoptosis assay by AO/EB and Hoechst 33342 staining methods}

To investigate the apoptotic effect induced by Ir and Lipo-Ir on A549 cells, the morphological changes in A549 cells were examined using Hoechst 33342 staining method and acridine orange/ethidium bromide (AO/EB) double staining technique. Compared to untreated cells, some typical apoptosis changes such as crenation, chromosome aggregation, apoptotic body, and fragmentation in chromatin morphology were observed in Ir and Lipo-Ir treatment groups (Figure 1A). In addition, $\mathrm{AO} / \mathrm{EB}$ staining was used to characterize cell apoptosis induced by Ir and Lipo-Ir. As demonstrated in Figure 1B, living cells appeared as weak green fluorescing nuclei and no significant apoptosis was detected in the control group. After treating with Ir and Lipo-Ir, the apoptotic cells showed bright green fluorescing nuclei, chromatin condensation, and nuclear shrinkage. In addition, the necrotic cells appearing with red fluorescence were found. All these morphological changes indicate that Lipo-Ir can effectively induce similar apoptosis in A549 cells.

\section{Cellular uptake}

Cellular uptake was investigated using IXM under the microscope after treatment with $8 \mu \mathrm{M}$ of Ir and Lipo-Ir for 0.5 or 2 h. Hoechst 33342 was also used to stain the cellular nuclei. As shown in Figure 2, in Ir-treated group, green fluorescence points were detected in the cellular cytoplasm, whereas a slightly weaker signal was recorded in the Lipo-Ir treatment group. With increasing time, bright green fluorescent points are observed, which indicate the cellular uptake increases in a time-dependent manner. These findings suggest that free Ir can be rapidly taken into cytoplasm during $30 \mathrm{~min}$, while the transport rate of Lipo-Ir into cells is slower than that of Ir. This may explain why the cytotoxicity induced by Ir is stronger than that induced by Lipo-Ir.

\section{Assay of location and the changes of MMP}

Mitochondria are potentially important therapeutic target. ${ }^{31}$ Lipophilic cations could be developed to target drugs to mitochondria in vivo. ${ }^{32,33}$ To further investigate the intracellular localization of free Ir and Lipo-Ir in the 

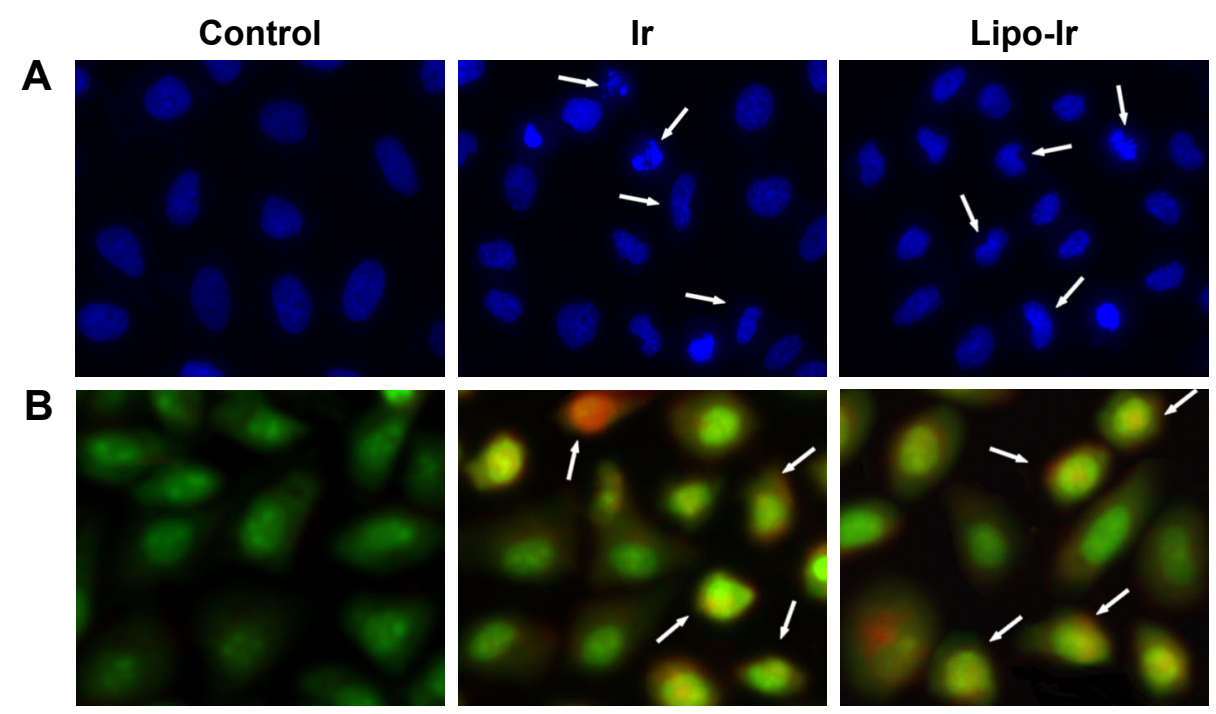

Figure I The morphology of apoptosis in A549 cells stained with Hoechst 33342 (A) and AO/EB (B) after treating with $3 \mu$ M Ir and equivalent Lipo-Ir for 24 h. Notes: The photographs were imaged using 40x objectives with DAPI (blue) or FITC (green) and Texas Red (red) filters. Arrows show the apoptotic body, chromosome aggregation, or apoptotic cells.

Abbreviations: Ir, iridium; Lipo-Ir, Ir-loaded PEGylated liposomes; PEG, polyethylene glycol; AO/EB, acridine orange/ethidium bromide.

mitochondria, MitoTracker ${ }^{\circledR}$ Deep Red FM was used as red fluorescent probe. As shown in Figure 3, the mitochondria were stained red, and after treatment of the cells with Ir or Lipo-Ir for $30 \mathrm{~min}, 2 \mathrm{~h}$, or $12 \mathrm{~h}$, the Ir and Lipo-Ir emitted green fluorescence. The merge of the red and the green fluorescence indicates that Ir and Lipo-Ir can enter into the mitochondria. The effect of Ir and Lipo-Ir on the changes of MMP $(\Delta \Psi m)$ was also investigated using JC-1 as fluorescent
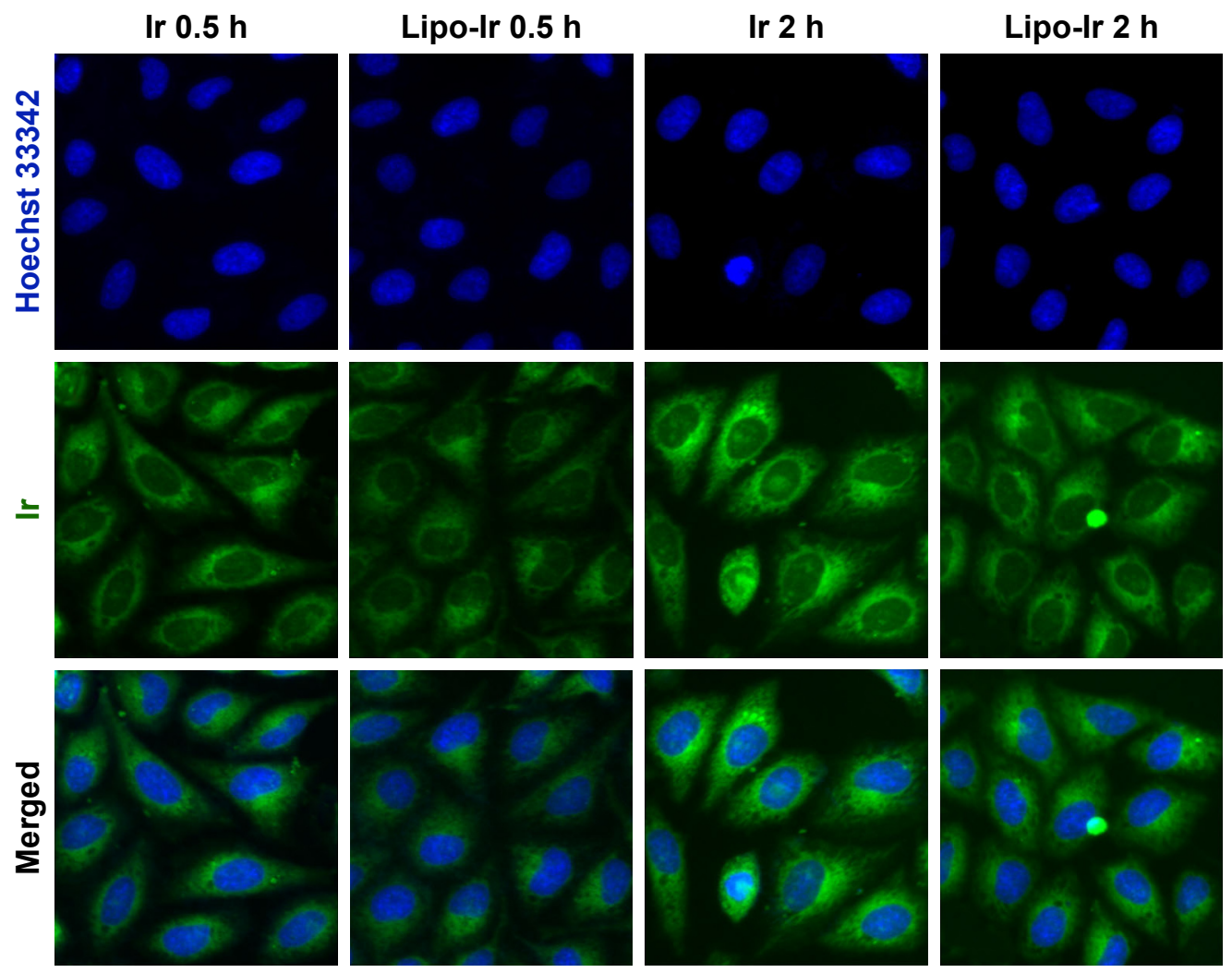

Figure 2 Cellular uptake of Ir and Lipo-Ir into A549 cells after treatment with $8 \mu \mathrm{M}$ Ir and equivalent Lipo-Ir (green) for 0.5 or $2 \mathrm{~h}$.

Note: Hoechst 33342 (blue) was also used to identify the cellular nuclei.

Abbreviations: Ir, iridium; Lipo-Ir, Ir-loaded PEGylated liposomes; PEG, polyethylene glycol. 


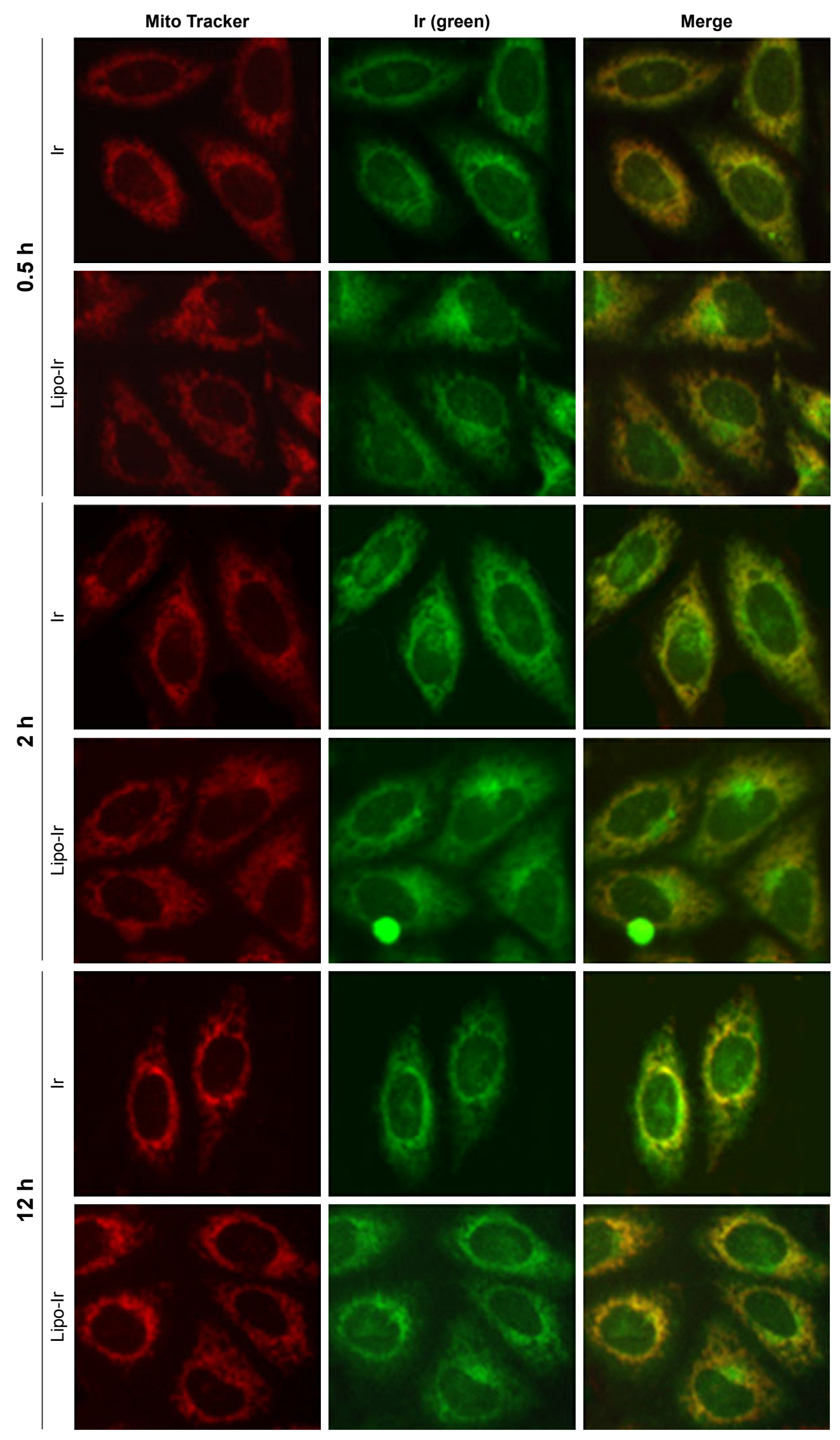

Figure 3 Assay of Ir and Lipo-Ir at the location of mitochondria.

Abbreviations: Ir, iridium; Lipo-Ir, Ir-loaded PEGylated liposomes; PEG, polyethylene glycol. 
probe. At high MMP, JC-1 forms an aggregation and emits red fluorescence; at low MMP, JC-1, existing as a monomer, emits green fluorescence. ${ }^{34}$ As shown in Figure 4A, in the control, JC-1 emits red fluorescence. After A549 cells were exposed to $3 \mu \mathrm{M}$ of Ir and Lipo-Ir or CCCP (positive control) for $24 \mathrm{~h}, \mathrm{JC}-1$ emits green fluorescence with little red fluorescence. The shift from red to green fluorescence shows that Ir and Lipo-Ir can result in dissipation of the MMP. To further compare the effect induced by Ir and Lipo-Ir on the changes in MMP, the ratio of red/green fluorescence was determined by the MateXpress6 software. As exhibited in Figure 4B, in the control, the ratio of red/green fluorescence was $1.72 \pm 0.16$. After treating with $\operatorname{Ir}(3 \mu \mathrm{M})$ and Lipo-Ir $(3 \mu \mathrm{M})$ for $24 \mathrm{~h}$, the ratios of red/green fluorescence were $0.69 \pm 0.06$ and $1.08 \pm 0.21$, respectively. The results demonstrate that Ir and Lipo-Ir can enter into the mitochondria and trigger collapse in the MMP.

\section{ROS detection}

Excess intracellular ROS is a potential element to destroy nucleic acid, lipid membranes, and organelles, which can activate apoptosis in cancer cells. ${ }^{35}$ The intracellular ROS levels were assayed by using DCFH-DA as fluorescent probe. It is well known that DCFH-DA can be converted into DCFH by intracellular esterase and DCFH can be oxidized by intracellular ROS to form a fluorescent DCF. ${ }^{36}$ As shown in Figure 5A, in the control, weak green fluorescence was observed. After being treated with $3 \mu \mathrm{M}$ of Ir or Lipo-Ir for $24 \mathrm{~h}$, a number of bright green fluorescence points were found. These findings suggest that Ir or Lipo-Ir enhances intracellular ROS levels. Additionally, the DCF fluorescent intensity was also determined by the MeteXpress6 software. As shown in Figure 5B, when A549 cells were treated with $3 \mu \mathrm{M}$ of Ir or Lipo-Ir for $24 \mathrm{~h}$, the cellular fluorescence intensities of DCF increased $\sim 2.79$ and 2.27 times than that in the control, respectively. The result exhibits that the intracellular ROS levels are significantly elevated by Ir and Lipo-Ir.

\section{Intracellular $\mathrm{Ca}^{2+}$ measurement}

Cellular $\mathrm{Ca}^{2+}$ overload is recognized as a cause of cytotoxicity and often plays a prominent role in $\mathrm{Ca}^{2+}$-dependent effectors such as the process of necrotic, apoptotic, and autophagic cell death. ${ }^{37,38}$ To monitor the changes in intracellular calcium, Fluo-3 AM was used as fluorescent probe. As shown in Figure 6A, a significant increase in the fluorescent intensity of Fluo-3 in A549 cells was observed after A549 cells were
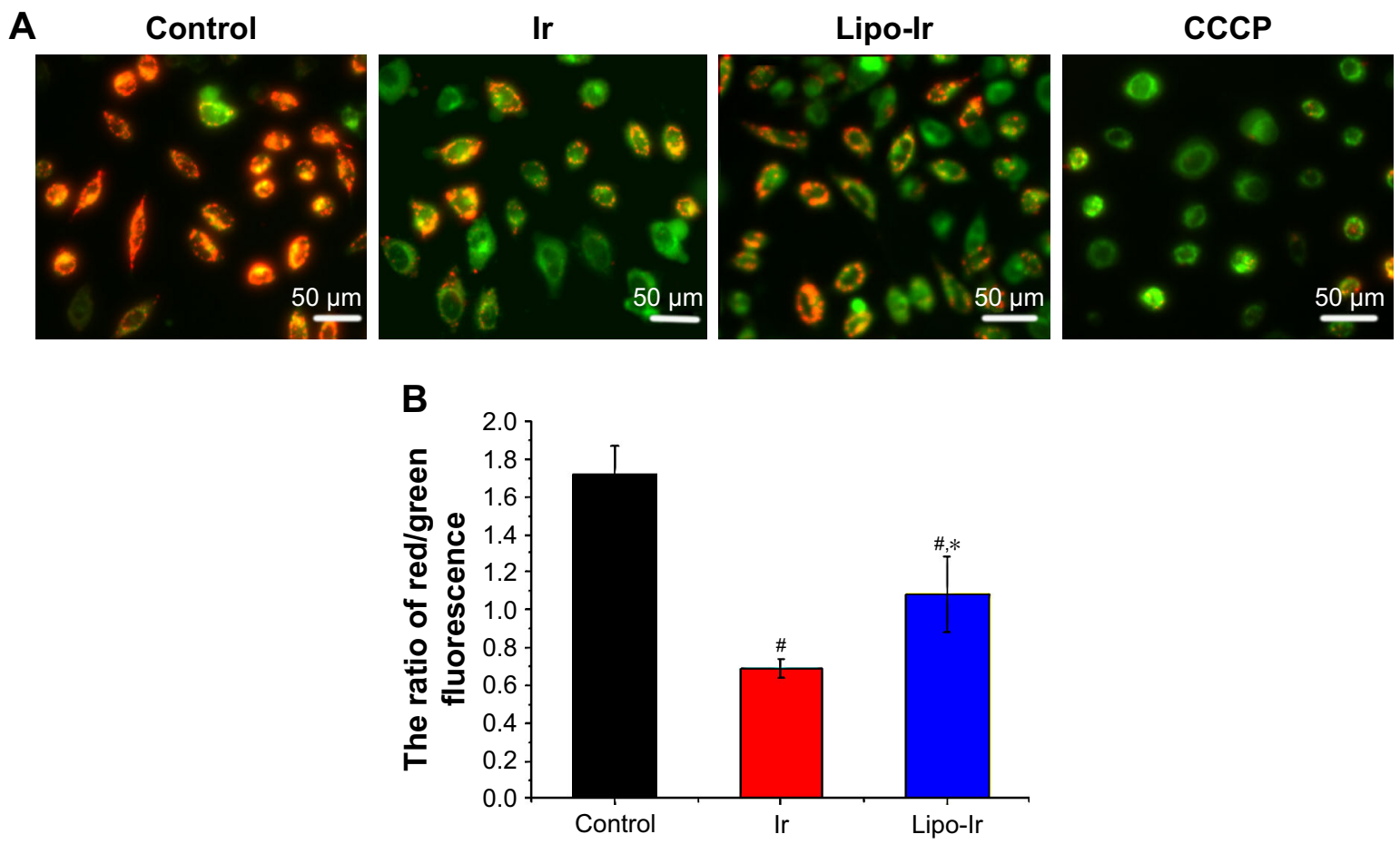

Figure 4 Assay of A549 cells mitochondrial membrane potential with JC-I staining method.

Notes: (A) The depolarization of mitochondrial membrane potential was observed (the magnification for the figure is $200 \times)$ after the cells were exposed to Ir ( $3 \mu \mathrm{M})$, equivalent Lipo-Ir for $24 \mathrm{~h}$, or CCCP (as positive control). (B) Quantitative analysis of ratio of red/green fluorescent intensity. Results of the red and green fluorescence are overlapped and expressed as mean \pm SD of triplicates. The Lipo-Ir-treated group exhibits significant difference compared with the Ir $(* P<0.05)$. The significant difference is observed among the treatment group and the blank control group $\left({ }^{\sharp} P<0.05\right)$.

Abbreviations: Ir, iridium; Lipo-Ir, Ir-loaded PEGylated liposomes; JC-I, 5,5',6,6'-tetrachloro-I, I',3,3'-tetraethylbenzimidazolcarbocyanine iodide; PEG, polyethylene glycol. 

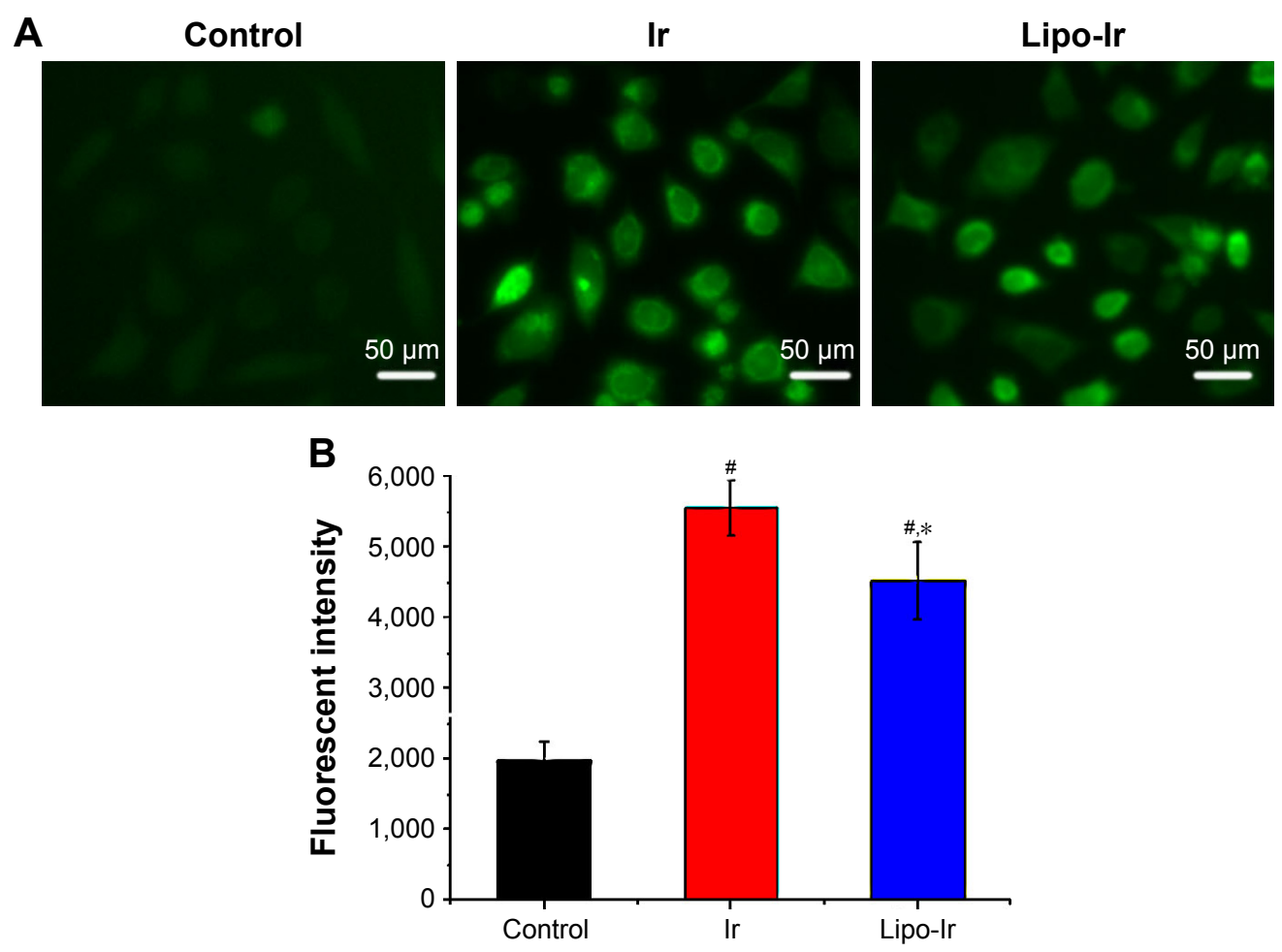

Figure 5 Analysis of the ROS level in A549 cells $(n=3)$.

Notes: (A) ROS generation in the cells exposed to Ir $(3 \mu \mathrm{M})$ and Lipo-Ir ( $\mu \mathrm{M}$ Ir-equivalent) for 24 h; results are representative of 30 randomly captured images (the magnification for the figure is $200 \times$ ). (B) Quantitative analysis of the DCF fluorescent intensity in A549 cells. Results are presented as mean \pm SD. $* P<0.05$ versus Ir; $\# P<0.05$ versus the blank control.

Abbreviations: DCF, dichlorofluorescein; Ir, iridium; Lipo-Ir, Ir-loaded PEGylated liposomes; PEG, polyethylene glycol; ROS, reactive oxygen species.

A

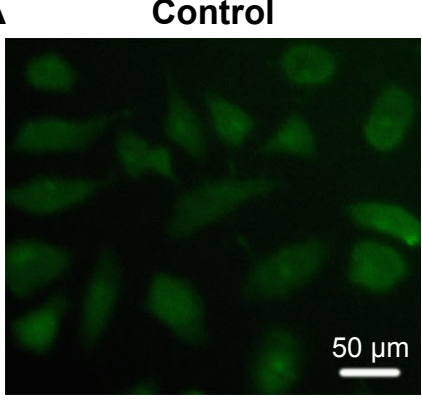

Ir

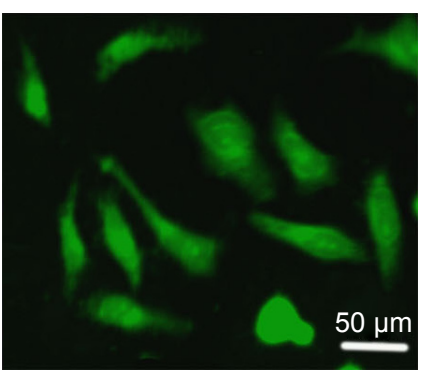

Lipo-Ir

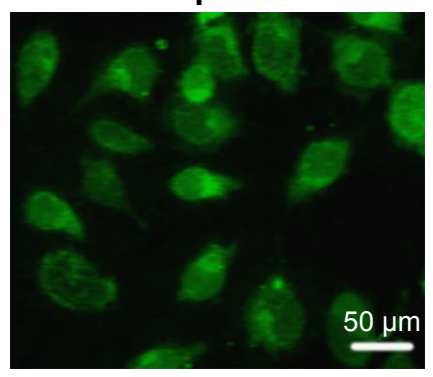

B

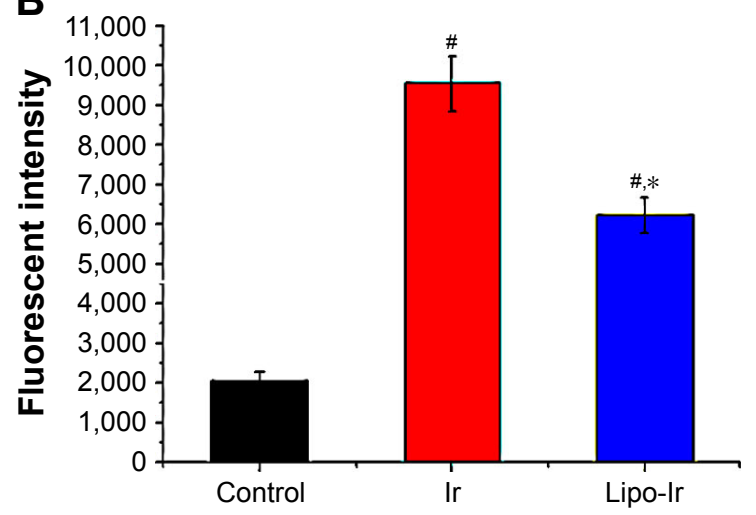

Figure 6 Determination of the levels of $\mathrm{Ca}_{i}{ }^{2+}$ in A549 cells using Fluo-3 AM as fluorescent probe.

Notes: (A) The $\mathrm{Ca}_{1}{ }^{2+}$ was monitored after being treated with $3 \mu \mathrm{M}$ Ir and equivalent Lipo-Ir for $24 \mathrm{~h}$. The magnification for the figure is $200 \times$. (B) Quantitative results of the fluorescent intensity of Fluo-3. Results are displayed as mean \pm SD $(n=3)$. $* P<0.05$ compared with Ir. ${ }^{*}<0.05$ compared with the blank control.

Abbreviations: Ir, iridium; Lipo-Ir, Ir-loaded PEGylated liposomes; PEG, polyethylene glycol. 
exposed to $3 \mu \mathrm{M}$ Ir and equivalent Lipo-Ir for $24 \mathrm{~h}$. The fluorescence intensity of Fluo-3 increased 4.5 times for Ir and 3.0 times for Lipo-Ir than that of control (Figure 6B). This result demonstrates that Ir and Lipo-Ir can enhance intracellular $\mathrm{Ca}^{2+}$ levels, which will lead to mitochondrial dysfunction and promote apoptosis. ${ }^{39}$

\section{Cell cycle arrest by flow cytometry}

The apoptosis of tumor cells is associated with cell cycle perturbation. ${ }^{40}$ To study the effects of Ir and Lipo-Ir on the blockage of cell cycle, the cell cycle arrest assay was performed by flow cytometry. As shown in Figure S5, in the control, the percentage at $\mathrm{G} 0 / \mathrm{G} 1$ phase was 41.96. After the treatment of A549 cells with $3 \mu \mathrm{M}$ of Ir or Lipo-Ir for $24 \mathrm{~h}$, the percentage at G0/G1 phase was 50.72 and 49.51, respectively. An increase of $8.76 \%$ for Ir and $7.55 \%$ for Lipo-Ir was observed. In addition, an increase of $14.31 \%$ for Ir and $11.43 \%$ for Lipo-Ir was found after A549 cells were treated with $5 \mu \mathrm{M}$ of $\mathrm{Ir}$ or Lipo-Ir for $24 \mathrm{~h}$, which was accompanied by a reduction in the percentage of the cells at $\mathrm{S}$ phase. These data indicate that Ir and Lipo-Ir inhibit the cell growth at G0/G1 phase.

\section{Conclusion}

In the present work, we have successfully encapsulated Ir into PEGylated liposomes with high EE of $94.71 \%$. The prepared Lipo-Ir has small size $(112.57 \pm 1.15 \mathrm{~nm})$ and spherical shape without any aggregation. Moreover, Lipo-Ir displays a good performance of physical stability, biocompatibility, and sustained effect. In the test, the hemolysis rate is $<5 \%$ in the RBCs treated with $93.75 \mu \mathrm{g} / \mathrm{mL}$ Lipo-Ir. However, when treated with Ir under the same concentration, it reaches up to $50 \%$. Additionally, the accumulative release for $96 \mathrm{~h}$ in the Lipo-Ir treatment group and the Ir-treated group is $47.17 \%$ and $91.75 \%$, respectively. The aforementioned results suggest that the Lipo-Ir is suitable for intravenous administration. Furthermore, Lipo-Ir can maintain the remarkable anticancer activity induced by the free Ir. In the assay of MMP, ROS, and intracellular $\mathrm{Ca}^{2+}$, Lipo-Ir induces a 1.6-fold decrease in MMP, a 2.27-fold increase in the ROS levels, and a 3.0-fold increase in intracellular calcium compared with the blank control group, which further promotes cell apoptosis. Moreover, Lipo-Ir has the blockage efficacy in $\mathrm{G}_{0} / \mathrm{G}_{1}$ phase of cell cycle. In conclusion, Lipo-Ir induces apoptosis in A549 cells through the ROSmediated mitochondrial dysfunction pathway and Lipo-Ir will be a promising approach for effective tumor therapy.

\section{Acknowledgment}

This study was financially supported by the National Nature Science Foundation of China (no 81403111).

\section{Disclosure}

The authors report no conflicts of interest in this work.

\section{References}

1. Jemal A, Ward EM, Johnson CJ, et al. Annual report to the nation on the status of cancer, 1975-2014, featuring survival. J Natl Cancer Inst. 2017; 109(9):1-22.

2. Momekov G, Ferdinandov D, Bakalova A, Zaharieva M, Konstantinov S, Karaivanova M. In vitro toxicological evaluation of a dinuclear platinum(II) complex with acetate ligands. Arch Toxicol. 2006;80(9):555-560.

3. Jung Y, Lippard SJ. Direct cellular responses to platinum-induced DNA damage. Chem Rev. 2007;107(5):1387-1407.

4. Wu Z, Mu J, Wang Q, et al. Hydroxyl and amino functionalized cyclometalated Ir(III) complexes: synthesis, characterization and cytotoxicity studies. J Organomet Chem. 2015;791:175-182.

5. Liu Z, Salassa L, Habtemariam A, Pizarro AM, Clarkson GJ, Sadler PJ Contrasting reactivity and cancer cell cytotoxicity of isoelectronic organometallic iridium (III) complexes. Inorg Chem. 2011;50(12):5777-5783.

6. Hisamatsu Y, Shibuya A, Suzuki N, et al. Design and synthesis of amphiphilic and luminescent tris-cyclometalated iridium(III) complexes containing cationic peptides as inducers and detectors of cell death via a calcium-dependent pathway. Bioconjug Chem. 2015;26(5):857-879.

7. You Y, Nam W. Photofunctional triplet excited states of cyclometalated Ir(III) complexes: beyond electroluminescence. Chem Soc Rev. 2012;41(21):7061-7084

8. Tang B, Wan D, Wang YJ, Yi QY, Guo BH, Liu YJ. An iridium (III) complex as potent anticancer agent induces apoptosis and autophagy in B16 cells through inhibition of the AKT/mTOR pathway. Eur J Med Chem. 2018;145:302-314.

9. Hart M, Acott S. Physical and chemical stability of Taxotere (docetaxel) one-vial $(20 \mathrm{mg} / \mathrm{ml})$ infusion solution following refrigerated storage. Ecancermedicalscience. 2010;4:202.

10. Weiszhár Z, Czúcz J, Révész C, Rosivall L, Szebeni J, Rozsnyay Z. Complement activation by polyethoxylated pharmaceutical surfactants: Cremophor-EL, Tween-80 and Tween-20. Eur J Pharm Sci. 2012;45(4):492-498.

11. van Zuylen L, Verweij J, Sparreboom A. Role of formulation vehicles in taxane pharmacology. Invest New Drugs. 2001;19(2):125-141.

12. Child KJ, Currie JP, Dis B, et al. The pharmacological properties in animals of CT1341-a new steroid anaesthetic agent. Br J Anaesth. 1971; 43(1):2-13.

13. Doebeli A, Michel E, Bettschart R, Hartnack S, Reichler IM. Apgar score after induction of anesthesia for canine cesarean section with alfaxalone versus propofol. Theriogenology. 2013;80(8):850-854.

14. Qu W, Zuo W, Li N, et al. Design of multifunctional liposomequantum dot hybrid nanocarriers and their biomedical application. J Drug Target. 2017;25(8):661-672.

15. Jone A. Liposomes: a short review. J Pharm Sci Res. 2013;5(9):181-183.

16. Kolate A, Baradia D, Patil S, Vhora I, Kore G, Misra A. PEG - a versatile conjugating ligand for drugs and drug delivery systems. $J$ Control Release. 2014;192:67-81.

17. Kohli AG, Kierstead PH, Venditto VJ, Walsh CL, Szoka FC. Designer lipids for drug delivery: from heads to tails. J Control Release. 2014;190: 274-287.

18. Allen TM. The use of glycolipids and hydrophilic polymers in avoiding rapid uptake of liposomes by the mononuclear phagocyte system. $A d v$ Drug Del Rev. 1994;13(3):285-309.

19. Immordino ML, Dosio F, Cattel L. Stealth liposomes: review of the basic science, rationale, and clinical applications existing and potential. Int J Nanomedicine. 2006;1(3):297-315.

20. Zhang C, Lai SH, Zeng CC, et al. The induction of apoptosis in SGC-7901 cells through the ROS-mediated mitochondrial dysfunction pathway by a Ir(III) complex. J Biol Inorg Chem. 2016;21(8):1047-1060.

21. Huang SL, MacDonald RC. Acoustically active liposomes for drug encapsulation and ultrasound-triggered release. Biochim Biophys Acta. 2004;1665(1-2):134-141. 
22. See E, Zhang W, Liu J, et al. Physicochemical characterization of asulacrine towards the development of an anticancer liposomal formulation via active drug loading: stability, solubility, lipophilicity and ionization. Int J Pharm. 2014;473(1-2):528-535.

23. Xiong H, Du S, Ni J, Zhou J, Yao J. Mitochondria and nuclei dualtargeted heterogeneous hydroxyapatite nanoparticles for enhancing therapeutic efficacy of doxorubicin. Biomaterials. 2016;94:70-83.

24. Cao Z, Cui Y, Nguyen HM, Jenkins DP, Wulff H, Pessah IN. Nanomolar bifenthrin alters synchronous $\mathrm{Ca}^{2+}$ oscillations and cortical neuron development independent of sodium channel activity. Mol Pharmacol. 2014;85(4):630-639.

25. Gregoriadis G, Leathwood PD, Ryman BE. Enzyme entrapment in liposomes. FEBS Lett. 1971;14(2):95-99.

26. Bhattacharjee S. DLS and zeta potential - what they are and what they are not? J Control Release. 2016;235:337-351.

27. Treuel L, Eslahian KA, Docter D, et al. Physicochemical characterization of nanoparticles and their behavior in the biological environment. Phys Chem Chem Phys. 2014;16(29):15053-15067.

28. Xiang B, Jia XL, Qi JL, et al. Enhancing sirNa-based cancer therapy using a new ph-responsive activatable cell-penetrating peptide-modified liposomal system. Int J Nanomedicine. 2017;12:2385-2405.

29. Kouchakzadeh H, Shojaosadati SA, Maghsoudi A, Vasheghani Farahani E. Optimization of PEGylation conditions for BSA nanoparticles using response surface methodology. AAPS PharmSciTech. 2010;11(3): 1206-1211.

30. Shen J, Kim HC, Wolfram J, et al. A liposome encapsulated ruthenium polypyridine complex as a theranostic platform for triple-negative breast cancer. Nano Lett. 2017;17(5):2913-2920.

31. Smith RA, Hartley RC, Cochemé HM, Murphy MP. Mitochondrial pharmacology. Trends Pharmacol Sci. 2012;33(6):341-352.
32. Smith RA, Hartley RC, Murphy MP. Mitochondria-targeted small molecule therapeutics and probes. Antioxid Redox Signal. 2011;15(12): 3021-3038.

33. Zielonka J, Joseph J, Sikora A, et al. Mitochondria-targeted triphenylphosphonium-based compounds: syntheses, mechanisms of action, and therapeutic and diagnostic applications. Chem Rev. 2017; 117(15):10043-10120.

34. Li S, Dong P, Wang J, et al. Icariin, a natural flavonol glycoside, induces apoptosis in human hepatoma SMMC-7721 cells via a ROS/JNKdependent mitochondrial pathway. Cancer Lett. 2010;298(2):222-230.

35. Pyle AM, Rehmann JP, Meshoyrer R, et al. Mixed-ligand complexes of ruthenium(II): factors governing binding to DNA. J Am Chem Soc. 1989;111(8):3051-3058.

36. Wrona M, Patel K, Wardman P. Reactivity of $2^{\prime}, 7^{\prime}$-dichlorodihydrofluorescein and dihydrorhodamine 123 and their oxidized forms toward carbonate, nitrogen dioxide, and hydroxyl radicals. Free Radic Biol Med. 2005;38(2):262-270.

37. Zhivotovsky B, Orrenius S. Calcium and cell death mechanisms: a perspective from the cell death community. Cell Calcium. 2011; 50(3):211-221.

38. Prevarskaya N, Ouadid-Ahidouch H, Skryma R, Shuba Y. Remodelling of $\mathrm{Ca}^{2+}$ transport in cancer: how it contributes to cancer hallmarks? Philos Trans R Soc Lond B Biol Sci. 2014;369(1638):20130097.

39. Farsinejad S, Gheisary Z, Ebrahimi Samani S, et al. Mitochondrial targeted peptides for cancer therapy. Tumor Biol. 2015;36(8):5715-5725.

40. Shapiro GI, Harper JW. Anticancer drug targets: cell cycle and checkpoint control. J Clin Invest. 1999;104(12):1645-1653. 


\section{Supplementary materials Materials and methods \\ Materials}

Tween-80 was obtained from Aladdin (Pharmaceutical grade; Shanghai, China). Acridine orange (AO), ethidium bromide (EB), propidium iodide (PI), RNAse A, and vitamin E were obtained from Sigma-Aldrich Co. (St Louis, MO, USA). 3-(4,5-Dimethylthiazole)-2,5-diphenltetraazolium bromide (MTT), 5,5',6,6'-tetrachloro-1,1',3,3'-tetraethylbenzimidazol carbocyanine iodide (JC-1), 2', $7^{\prime}$-dichlorodihydrofluorescein diacetate (DCFH-DA), Fluo-3 AM, and Hoechst 33342 were purchased from Shanghai Beyotime Institute of Biotechnology (Shanghai, China). One percentage penicillin-streptomycin and MitoTracker ${ }^{\circledR}$ Deep Red FM were obtained from Thermo Fisher Scientific (Waltham, MA, USA). All other reagents were analytical or chromatographic grade.

RE-SZAA rotary vacuum evaporator (Shanghai YaRong Biochemical Instrument Factory, Shanghai, China), Scientz-IID cell sonicator (Ningbo Scientz Biotechnology Co., Ltd., Ningbo, Zhejiang, China), Optima L-100XP Preparative ultracentrifuge (Beckman Coulter, Brea, CA, USA), Hitachi H-7650 transmission electron microscopy (TEM; Hitachi Ltd. Tokyo, Japan), ImageXpress ${ }^{\circledR}$ High Content Screening System (IXM; Molecular Devices LLC, Sunnyvale, CA, USA), FACS Calibur flow cytometer (Beckman Dickinson, Franklin Lakes, NJ, USA), and cell culture plates (Costar; Corning Incorporated, Corning, NY, USA) were used.

\section{HPLC analysis}

Iridium (Ir) was determined using a HPLC system (1200 LC; Agilent Technologies, Santa Clara, CA, USA). The samples were analyzed using a $\mathrm{C}_{18}$ column (5 $\mu \mathrm{m}, 4.6 \times 250 \mathrm{~mm}$, Diamonsil). The mobile phase was acetonitrile and $0.02 \mathrm{M}$ sodium acetate buffer containing $0.06 \mathrm{M} \mathrm{NaClO}_{4}$ (final $\mathrm{pH} 4.65$ ) (70:30, v/v). The flow rate, injection volume, and detection wavelength were set as $1.0 \mathrm{~mL} / \mathrm{min}, 20 \mu \mathrm{L}$, and $260 \mathrm{~nm}$, respectively.
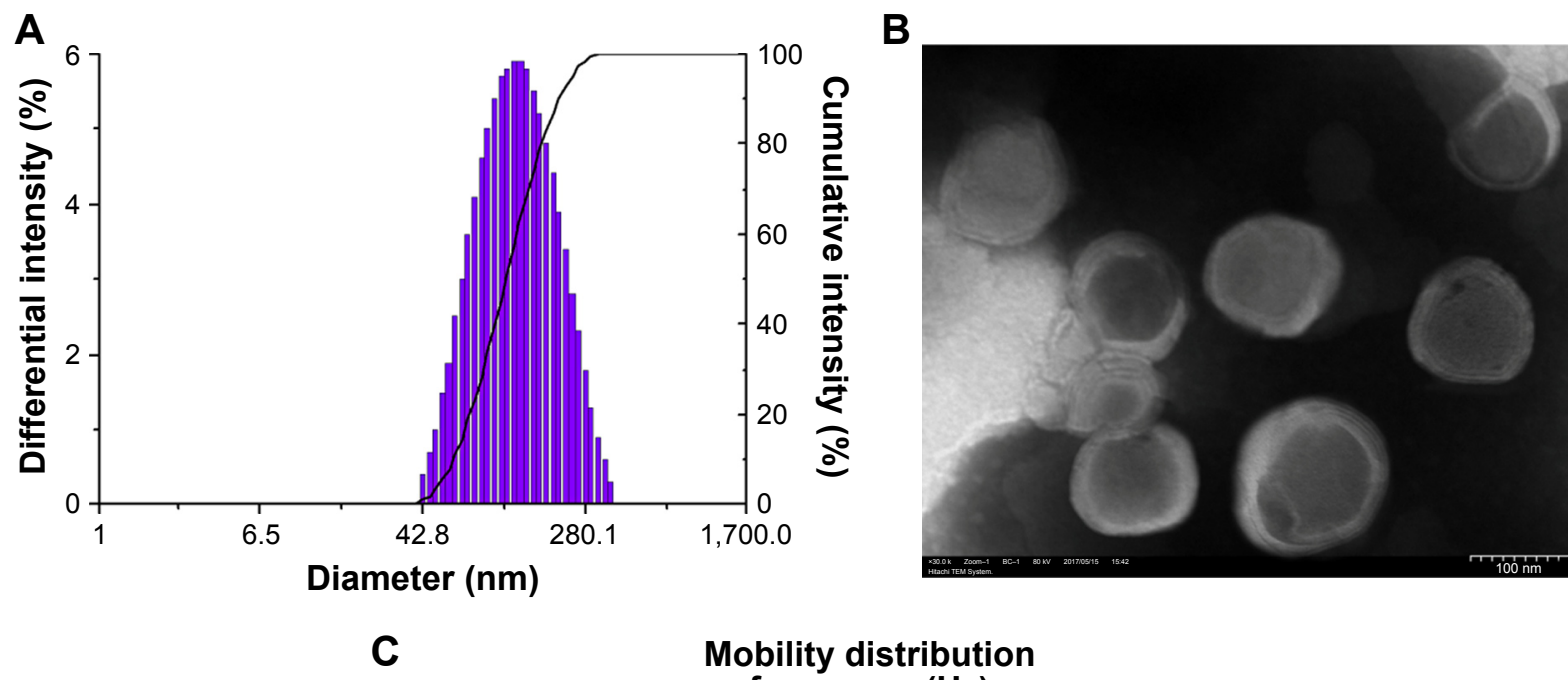

Mobility distribution

frequency $(\mathrm{Hz})$

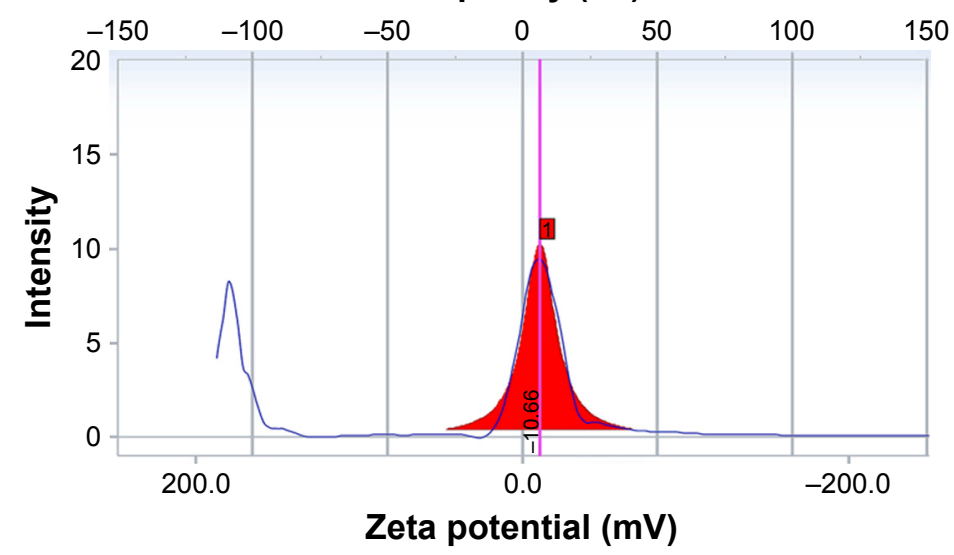

Figure SI Characterization of Lipo-Ir.

Notes: (A) Size distribution, (B) TEM image, and (C) zeta potential.

Abbreviations: Ir, iridium; Lipo-Ir, Ir-loaded PEGylated liposomes; PEG, polyethylene glycol; TEM, transmission electron microscopy. 
Table SI Characterization parameters of liposomes $(n=3)$

\begin{tabular}{llllll}
\hline Liposomes & Size $(\mathbf{n m})$ & PDI & Zeta potential $(\mathbf{m V})$ & EE (\%) & DL (\%) \\
\hline Blank-Lipo & $92.80 \pm 3.08$ & $0.13 \pm 0.03$ & $-7.03 \pm 0.12$ & - & - \\
Lipo-Ir & $116.57 \pm 1.15$ & $0.19 \pm 0.02$ & $-10.66 \pm 0.6 \mathrm{I}$ & $94.7 \mathrm{I} \pm 3.2 \mathrm{I}$ & $4.7 \mathrm{I} \pm 0.4 \mathrm{I}$ \\
\hline
\end{tabular}

Abbreviations: Blank-Lipo, blank PEGylated liposomes; DL, drug loading; EE, encapsulation efficiency; Ir, iridium; Lipo-Ir, Ir-loaded PEGylated liposomes; PDI, polydispersity index; PEG, polyethylene glycol.

Table S2 Physical stability of Lipo-Ir $(n=3)$

\begin{tabular}{lll}
\hline Time (days) & Size $(\mathbf{n m})$ & EE $(\%)$ \\
\hline 0 & $|I| 6.57 \pm I .15$ & $94.7 I \pm 3.21$ \\
15 & $1 \mid 7.60 \pm I .59$ & $94.01 \pm 4.20$ \\
30 & $1 \mid 8.75 \pm 2.12$ & $95.24 \pm 3.95$ \\
60 & $\mid I 8.92 \pm 4.14$ & $94.93 \pm 4.98$
\end{tabular}

Abbreviations: EE, encapsulation efficiency; Ir, iridium; Lipo-Ir, Ir-loaded PEGylated liposomes; PEG, polyethylene glycol.

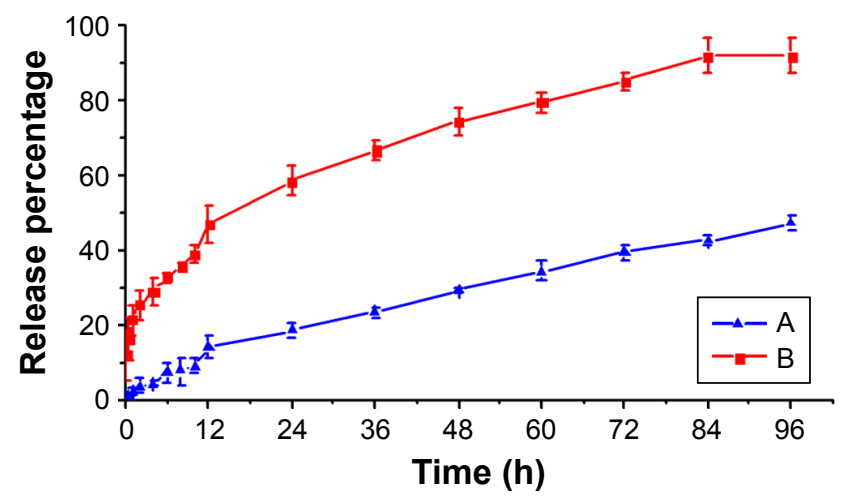

Figure S2 In vitro release profiles of Ir from Lipo-Ir (A) and Ir (B). Note: Bars represent \pm SD $(n=3)$.

Abbreviations: Ir, iridium; Lipo-Ir, Ir-loaded PEGylated liposomes; PEG, polyethylene glycol.

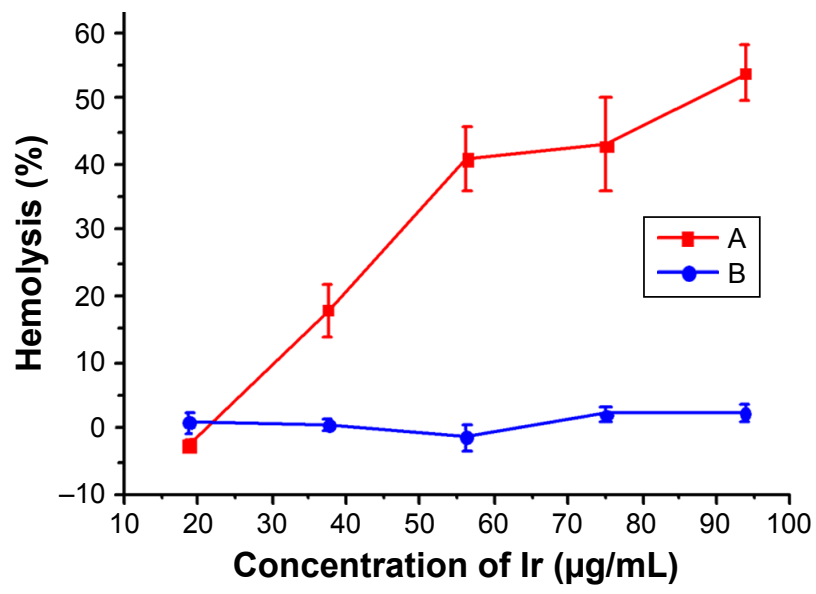

Figure S3 Hemolysis percentages of $\operatorname{Ir}(\mathbf{A})$ and Lipo-Ir (B).

Note: Bars represent \pm SD $(n=3)$.

Abbreviations: Ir, iridium; Lipo-Ir, Ir-loaded PEGylated liposomes; PEG, polyethylene glycol.
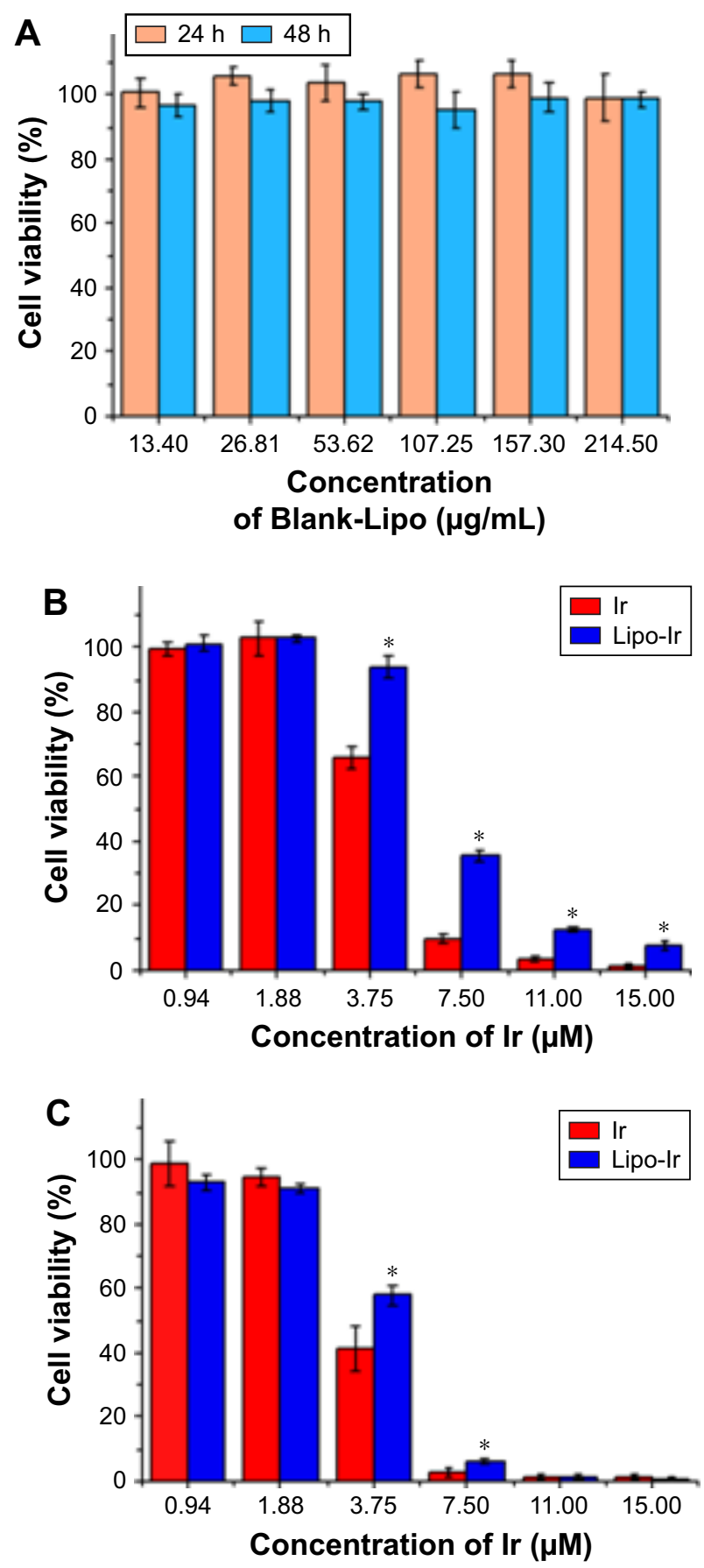

Figure S4 Evaluation of cell viability (MTT).

Notes: (A) Viability of A549 cells incubated with various concentrations of BlankLipo for 24 and $48 \mathrm{~h}$. Viability of A549 cells incubated with various concentrations of Ir or Lipo-Ir for $24 \mathrm{~h}$ (B) or $48 \mathrm{~h}$ (C). Results are presented as the mean \pm SD of triplicates. ${ }^{*} P<0.05$ compared with Ir.

Abbreviations: Blank-Lipo, blank PEGylated liposomes; Ir, iridium; Lipo-Ir, Ir-loaded PEGylated liposomes; MTT, 3-(4,5-dimethylthiazole)-2,5-diphenltetraazolium bromide; PEG, polyethylene glycol. 

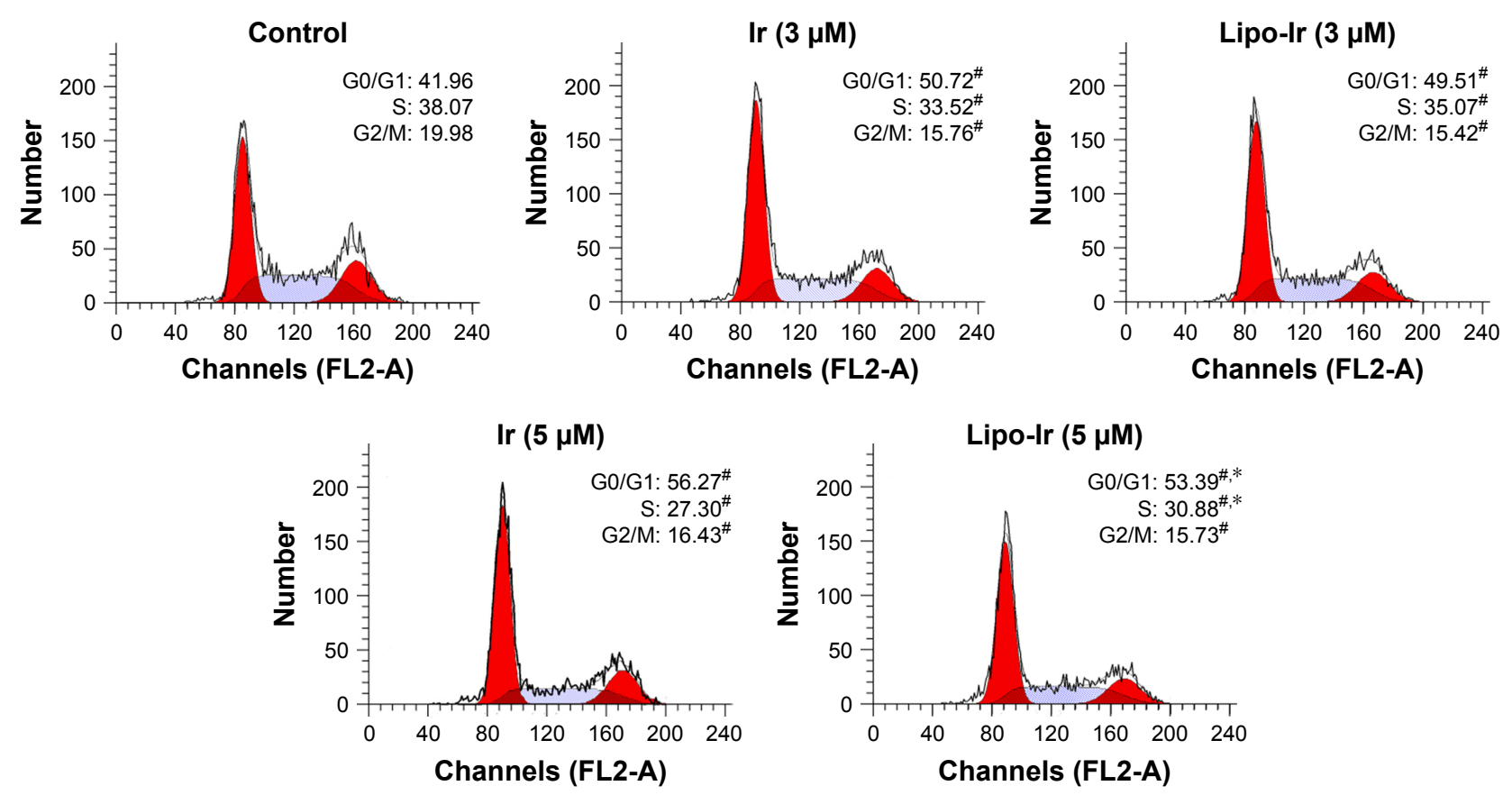

Figure S5 Cell cycle profiles in A549 cells were determined by flow cytometry after incubating with Ir (3 or $5 \mu \mathrm{M})$ and equivalent Lipo-Ir for 24 h. Notes: $* P<0.05$ compared with Ir (between same concentrations). ${ }^{P}<0.05$ compared with control.

Abbreviations: Ir, iridium; Lipo-Ir, Ir-loaded PEGylated liposomes; PEG, polyethylene glycol.

\section{Publish your work in this journal}

The International Journal of Nanomedicine is an international, peerreviewed journal focusing on the application of nanotechnology in diagnostics, therapeutics, and drug delivery systems throughout the biomedical field. This journal is indexed on PubMed Central, MedLine, CAS, SciSearch ${ }^{\circledR}$, Current Contents ${ }^{\circledR} /$ Clinical Medicine,
Journal Citation Reports/Science Edition, EMBase, Scopus and the Elsevier Bibliographic databases. The manuscript management system is completely online and includes a very quick and fair peer-review system, which is all easy to use. Visit http://www.dovepress.com/ testimonials.php to read real quotes from published authors. 